\title{
Glutathione peroxidase overexpression causes aberrant ERK activation in neonatal mouse cortex after hypoxic preconditioning
}

\author{
Delphine Autheman', R. Ann Sheldon², Nondini Chaudhuri', Sebastian von Arx' ', Corinne Siegenthaler', Donna M. Ferriero ${ }^{2,3}$ \\ and Stephan Christen ${ }^{1,2}$
}

BACKGROUND: Preconditioning of neonatal mice with nonlethal hypoxia (HPC) protects the brain from hypoxic-ischemic $(\mathrm{HI})$ injury. Overexpression of human glutathione peroxidase 1 (GPx1), which normally protects the developing murine brain from HI injury, reverses HPC protection, suggesting that a certain threshold of hydrogen peroxide concentration is required for activation of HPC signaling.

METHODS: Activation (phosphorylation) of extracellularregulated kinase (ERK) $1 / 2$ and Akt, and induction of hypoxiainducible factor (HIF)- $1 \alpha$ were assessed in the cortex, one of the main structures affected by $\mathrm{HI}$ and protected by HPC, at different time points after reoxygenation in wild-type (WT) and GPx1overexpressing animals.

RESULTS: GPX1 overexpression prevented both the global and nuclear increase in activated ERK at $0.5 \mathrm{~h}$ after HPC and caused a significant decrease in phospho-ERK (pERK)/ERK levels at $24 \mathrm{~h}$ after HPC. In contrast, HIF-1 $\alpha$ induction at the end of hypoxia was unaffected by GPX1 overexpression. In the cortex of preconditioned WT animals, enhanced pERK staining was primarily observed in neurons and to a lower extent in astrocytes and endothelial cells, with a nuclear prominence.

CONCLUSION: Aberrant activation of ERK probably explains the paradoxical reversal of HPC protection by GPX1 overexpression. The results identify hydrogen peroxide as an important mediator of neuroprotective ERK signaling.

P erinatal asphyxia and other birth complications can lead to hypoxic-ischemic (HI) injury to the neonatal brain. Preconditioning is a stimulus that triggers endogenous neuroprotective mechanisms in the brain and thereby provides protection from subsequent injury (1). In neonatal mice, a brief episode of systemic hypoxia is an effective stimulus for triggering neuroprotective mechanisms that lead to a reduction in HI brain injury (2). Studying the mechanisms involved in hypoxic preconditioning (HPC) provides a unique opportunity to understand endogenous neuroprotective processes that could also be important for the injured brain (1).

We have previously shown that $\mathrm{HI}$ in neonatal mice is associated with a marked decrease in glutathione peroxidase (GPx) activity
$24 \mathrm{~h}$ after HI, while catalase activity remains unchanged (3). This led us to investigate whether overexpression of human GPx 1 (GPx1) protects the neonatal brain from HI injury (4). Similar to adult animals after focal ischemia (5), GPx1-overexpressing mice have reduced histological brain injury after $\mathrm{HI}$, indicating that oxidative stress is a critical mediator of the injury response after neonatal HI. The protection afforded by GPxl overexpression is associated with increased GPx activity and reduced hydrogen peroxide accumulation after HI (2). GPxl overexpression also reduces $\mathrm{HI}$ injury in neonatal mice overexpressing human superoxide dismutase 1 (6), which have been shown to accumulate higher levels of hydrogen peroxide after $\mathrm{HI}$ (3) and present with greater damage as compared with nontransgenic wild-type (WT) littermates (7). These results clearly show that hydrogen peroxide plays an important role in neonatal $\mathrm{HI}$ injury and highlight the importance of GPx activity in removing hydrogen peroxide during HI. Paradoxically, however, we recently found that GPx1 overexpression reverses the neuroprotection afforded by HPC, which by itself does not affect GPx activity (2). This finding suggests that a certain threshold of hydrogen peroxide may be required during the preconditioning phase for the development of tolerance toward subsequent injury.

Development of tolerance by HPC is a time-dependent process that requires activation of transcriptional events to achieve maximum protection. Although much emphasis has been given to hypoxia-inducible factor $1 \alpha(\mathrm{HIF}-1 \alpha)$ as a mediator of this response, there is evidence that activation of extracellular signal-regulated kinase 1/2 (ERK1/2) or Akt may also be important in this process $(8,9)$. Thus, using oxygen/glucose deprivation to mimic ischemia in vivo, it was shown that inhibition of the ERK1/2 pathway abolished the protective effect of an ischemic preconditioning stimulus on the survival of isolated cortical rat neurons (10). More important, a study in neonatal rats showed that cortical ERK1/2 was transiently activated during the HPC phase, and inhibition of the ERK pathway with the mitogen-activated protein kinase kinase (MEK)1/2 inhibitor U0126 reversed HPC protection (11). The role of Akt activation in HPC protection in vivo is more controversial, as preconditioning is often not accompanied by activation of Akt $(11,12)$. 
Here, we assessed the temporal changes in the activation (phosphorylation) of ERK1/2 and Akt and the induction of HIF-1 $\alpha$ in the cortex of mice subjected to HPC, and determined how these pathways are affected by GPx1 overexpression, as these pathways have been shown to be subject to redox control (13-15). We focused on the cortex because it is one of the main structures affected by HI in neonatal mice (16); it is protected by HPC (2) and a comparison with previously published data can be drawn (11). We show that HPC is associated with transient activation of ERK1/2 but not Akt in the cortex of WT animals, and that this activation occurs in neurons, endothelial cells, and astrocytes in vulnerable regions and is associated with nuclear translocation. Finally, GPx1 overexpression blocked this activation and nuclear translocation of ERK, whereas it had no effect on the induction of HIF-1 $\alpha$. These results show that ERK activation in the murine brain by HPC is redox-dependent and provides evidence that aberrant ERK activation is responsible for the paradoxical reversal of HPC protection in GPx1-overexpressing mice.

\section{RESULTS}

HPC in Neonatal Mice Leads to Transient Activation of ERK1/2 and Induction of HIF- $1 \alpha$ but Not Activation of Akt in the Cerebral Cortex

To investigate which signaling pathways previously implicated in HPC protection could be affected by GPx1 overexpression, postnatal day $6 \mathrm{WT}$ animals were exposed to $45 \mathrm{~min}$ of global hypoxia ( $8 \%$ oxygen), and cortex tissue was analyzed for phospho-ERK (pERK) 1/2, phospho-Akt (pAkt), and HIF-1 $\alpha$ at different time points after reoxygenation, covering the whole preconditioning phase between 0 and $24 \mathrm{~h}$ posthypoxia (Figure 1a). Although phosphorylation of both ERK1 and ERK2 transiently and rapidly increased after reoxygenation, peaking at $0.5 \mathrm{~h}$ posthypoxia $(P<$ $0.05)$ and declining to prehypoxic levels after $24 \mathrm{~h}$ of reoxygenation, phosphorylation of Akt at Ser473 remained unchanged over this period (Figure 1b). Total protein levels of the two kinases remained unchanged over time (data not shown). HIF-1 $\alpha$ was markedly induced at the end of hypoxia ( $0 \mathrm{~h}$ posthypoxia) and was rapidly degraded again upon reoxygenation.

Overexpression of GPx1 Leads to Aberrant HPC-Mediated Activation of ERK1/2 and Nuclear Accumulation of pERK but Has No Effect on HIF- $1 \alpha$ Induction

To study the effect of GPx1 overexpression on HPC-induced ERK1/2 activation, we first analyzed the kinetics of ERK phosphorylation in GPx1 transgenic (tg) as compared with that in WT littermates (Figure 2a). Phosphorylated levels of ERK1 and ERK2 were combined and normalized to total ERK, given that HPC-induced activations of ERK1 and ERK2 in WT animals were similar (cf. Figure 1b). For easier comparison of the relative change in ERK phosphorylation in the two strains and because pERK/ERK levels in GPx1 naive mice did not significantly differ from those in WT naive mice $(108.0 \pm 14.3 \%, P>$ 0.05 by $t$-test), data were expressed relative to the corresponding naive controls. The kinetics of ERK phosphorylation in GPx1 tg mice was different from that in WT littermates $(P<0.01$ by twoway ANOVA). Thus, exposure of GPx1 tg mice to HPC did not

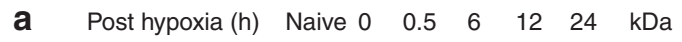
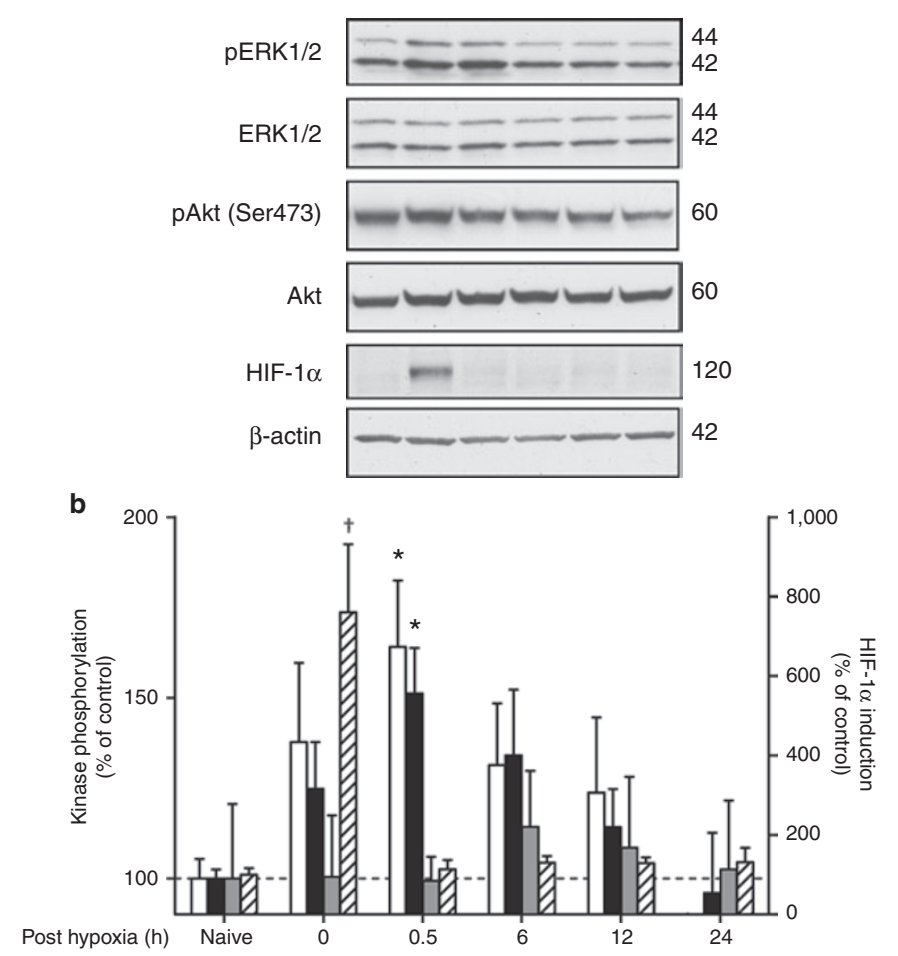

Figure 1. Hypoxic preconditioning transiently activates extracellularregulated kinase (ERK) in neonatal brain cortex. Postnatal day 6 mice were exposed to $45 \mathrm{~min}$ of global hypoxia ( $8 \%$ oxygen) and killed at various time points ( $0-24 \mathrm{~h}$ ) after reoxygenation. Animals exposed to normoxia served as baseline controls (naive). Phosphorylation of ERK1 (white bars), ERK 2 (black bars), and Akt (gray bars), and hypoxia-inducible factor (HIF)-1 $\alpha$ stabilization (hatched bars) were analyzed in cortical homogenates by western blotting as described in Methods. (a) Representative blots for a complete time course of one set of animals. (b) Bar graph shows a summary of the results from densitometric analysis of bands from $n=8$ (phospho-ERK/ERK), $n=5$ (pAkt/Akt), and $n=3$ (HIF-1 $\alpha$ ) independent sets of animals. The level of phosphoprotein is expressed relative to total protein and to that of wild-type naive mice $(=100 \%) \pm$ SEM. ${ }^{*} P<0.05$, $+P<0.001$, by one-way ANOVA.

cause transient ERK activation as in WT littermates $\left({ }^{\star} P<0.05\right.$ at $0.5 \mathrm{~h}$ posthypoxia), and ERK phosphorylation levels in GPx1 tg mice even appeared to drop below baseline at $24 \mathrm{~h}$ posthypoxia, in contrast to the normalization to prehypoxia levels at this time point in WT littermates (Figure $2 \mathbf{b}$ ). To corroborate the results obtained with the time course analysis, samples from $0.5 \mathrm{~h}$ and $24 \mathrm{~h}$ posthypoxia time points were analyzed separately (Figure $2 \mathrm{~b}, \mathrm{c}$ ). The results from these analyses confirm that ERK is significantly activated at $0.5 \mathrm{~h}$ after HPC in WT but not in GPx1 tg mice (Figure 2b) (pERK/ERK levels in GPx1 naive mice did not significantly differ from those in WT naive mice $(135.6 \pm 21.3 \%, P>0.05$ by $t$-test) $)$ and show that ERK phosphorylation levels at $24 \mathrm{~h}$ posthypoxia are significantly depressed in GPx1 tg mice as compared with corresponding WT littermates (Figure 2c) (pERK/ERK levels in GPx1 naive mice did not significantly differ from those in WT naive littermates $(92.9 \pm 18.5 \%, P>0.05$ by $t$-test $)$ ).

Upon activation of cytosolic ERK by phosphorylation, activated ERK moves to the nucleus and regulates the activity of 
a

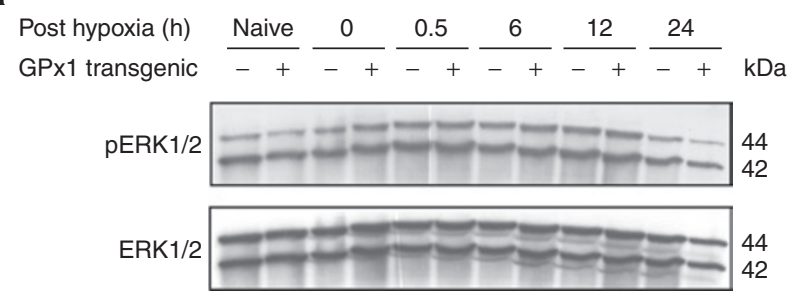

b

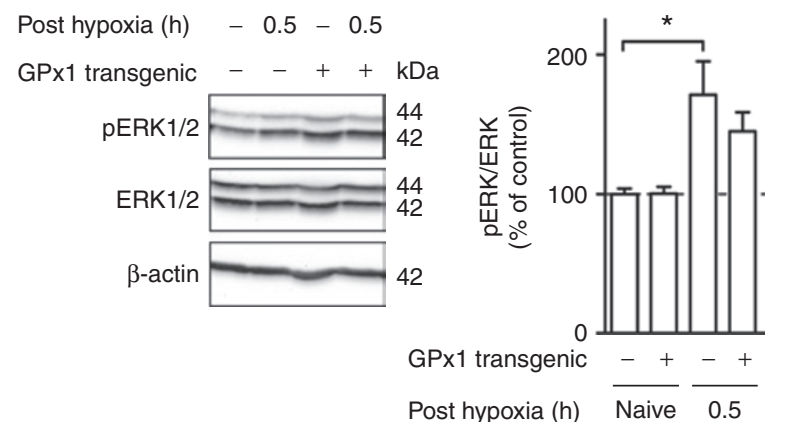

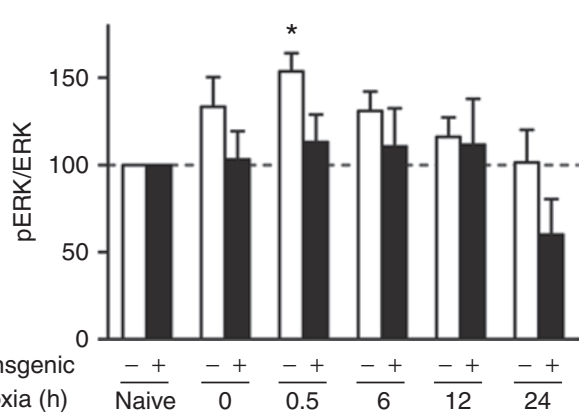

C

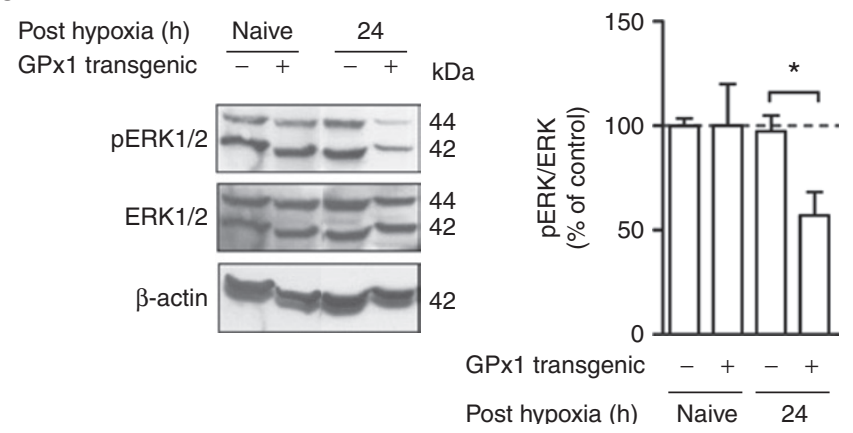

Figure 2. Overexpression of glutathione peroxidase 1 (GPx1) prevents hypoxic preconditioning (HPC)-mediated activation of cortical extracellular-regulated kinase (ERK). Phosphorylation of ERK was analyzed in cortical homogenates from wild-type (WT, white bars) and GPx1-overexpressing (black bars) animals exposed to preconditioning hypoxia at various time points after reoxygenation by western blotting. (a) Representative western blot of a complete time course of WT versus GPx1 transgenic (black) animals. Bar graph shows a summary of the results from densitometric analysis of bands from $n \geq 5$ independent sets of animals. Phospho-ERK is expressed relative to ERK and to that of respective naive mice $(=100 \%) \pm S E M\left({ }^{*} P<0.05\right.$, one-way ANOVA). (b) Representative western blot of samples taken $0.5 \mathrm{~h}$ after reoxygenation. Bar graph represents the mean \pm SEM of $n=10-11$ animals $\left({ }^{*} P<0.05\right.$, by one-way ANOVA) relative to respective naive controls. (c) Representative western blot of samples analyzed $24 \mathrm{~h}$ after HPC. Bar graph shows summary of densitometrically analyzed data from $n=5$ animals \pm SEM $(* P<0.05$ by one-way ANOVA) relative to respective naive controls.

several transcription factors involved in cell survival and proliferation (17). To see whether HPC causes an increase in activated ERK in the nucleus, cytosolic and nuclear extracts of cortex tissue were analyzed by western blotting (Figure 3a). Although preconditioning had no effect on the cytosolic or nuclear levels of ERK (ERK/ $\beta$-actin and ERK/HIF-1 $\beta$ ratios, respectively, data not shown), HPC caused a significant increase in nuclear pERK/ERK in WT animals but not in GPx1 overexpressors. Cytosolic levels were unaffected by HPC in both WT and GPx1 tg mice. Cytosolic pERK/ERK levels in GPx naive mice did not significantly differ from those in WT naive littermates (130.6 \pm $27.3 \%, P>0.05$ by $t$-test). In contrast to the lack of increased activated ERK in the nucleus in GPx1 tg animals, HIF- $1 \alpha$ induction at $0 \mathrm{~h}$ posthypoxia was unaffected by GPxl overexpression (Figure $3 \mathrm{~b}$ ). Thus, GPx overexpression selectively prevents the HPC-mediated nuclear increase in activated ERK.

\section{HPC-Induced Activation of ERK in the Cortex Occurs Mainly in Neurons}

To identify the cell type(s) responsible for the increased cortical ERK activation observed in the western blot experiments, brain sections from animals killed at $0.5 \mathrm{~h}$ posthypoxia were costained for pERK $1 / 2$ and the cellular markers neuronal nuclear antigen (for mature neurons), glial fibrillary acidic protein (for astrocytes), isolectin B4 (for endothelial cells, and microglia), or 2', $3^{\prime}$-cyclic nucleotide $3^{\prime}$-phosphodiesterase (for oligodendrocytes) and analyzed by immunofluorescence (Figure 4). At $0.5 \mathrm{~h}$ of reoxygenation, pERK staining was markedly induced in pyramidal neurons of cortical layer V (Figure $4 b, d$ ). Staining was predominantly nuclear, as judged by overlap with neuronal nuclear antigen (Figure 4d) and 4', 6'-diamidino-2-phenylindole (data not shown), in line with the data obtained with nuclear extracts. Overexpression of GPx1 prevented the increase in pERK staining in neurons (Figure 4c). In preconditioned WT animals, increased activated ERK was also found in astrocytes between subcortical fiber tracts and layer V (Figure 4e) and in parenchymal blood vessels (Figure 4f), although to a lesser extent than in neurons. This increase was also prevented by GPxl overexpression. pERK staining was also observed in oligodendrocytes of the corpus callosum (Figure $4 \mathrm{~g}$ ) but was independent of preconditioning and GPx1 overexpression. pERK staining in the hippocampus (another major structure affected by HI) was also increased by HPC, but to a lesser extent.

\section{DISCUSSION}

In this study, we show that the paradoxical loss of protection from HPC in GPx1 tg mice (2) is associated with aberrant ERK1/2 activation in the cortex. Thus, although HPC caused a transient increase of activated ERK at $0.5 \mathrm{~h}$ after reoxygenation, which led to nuclear accumulation of activated ERK, no significant increase was observed in GPx1 overexpressors. Furthermore, pERK/ERK levels in GPx1 overexpressors were 
a
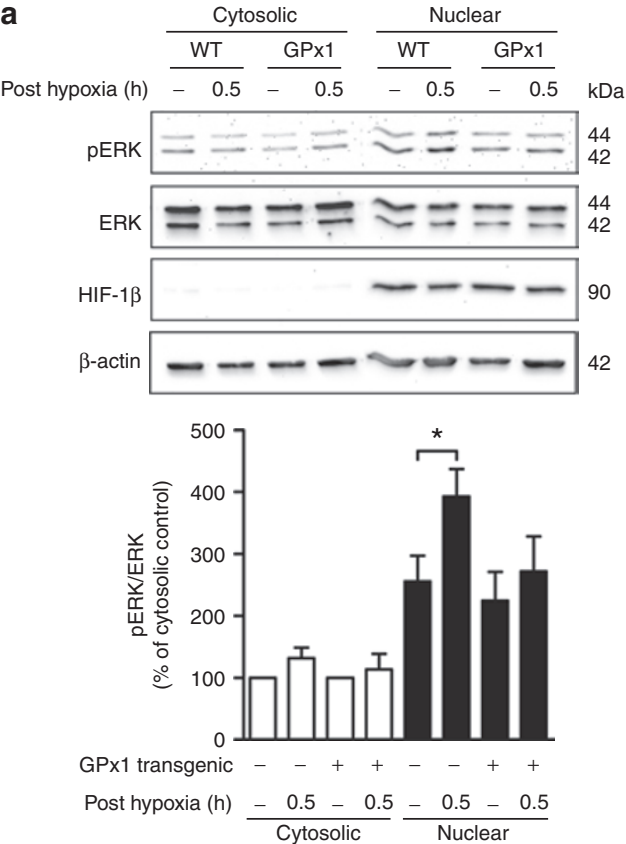

b
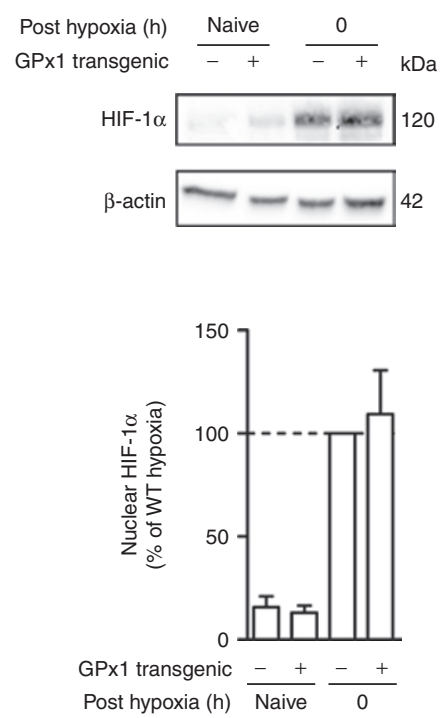

Figure 3. Overexpression of glutathione peroxidase 1 (GPx1) prevents nuclear accumulation of activated (a) extracellular-regulated kinase (ERK) but not of (b) hypoxia-inducible factor (HIF)- $1 \alpha$. (a) Nuclear (black bars) and cytosolic (white bars) extracts prepared at $0.5 \mathrm{~h}$ after reoxygenation were analyzed for phospho-ERK (pERK) and total ERK by western blotting. HIF-1 $\beta$ was used as a nuclear marker protein. (Top) Representative western blot of one set of samples. (Bottom) Bar graph shows a summary of the results from densitometric analysis of bands from $n=5$ independent sets of animals. Data are expressed relative to cytosolic level of $p E R K / E R K$ in respective naive controls $(=100 \%) \pm S E M .{ }^{*} P<0.05$, by one-way ANOVA. (b) Nuclear extracts prepared at $0 \mathrm{~h}$ after reoxygenation were analyzed for HIF-1 $\alpha$ by western blotting. (Top) Representative western blot of HIF- $1 \alpha$ analysis in nuclear extracts. (Bottom) Bar graph shows summary of results from densitometric analysis of HIF- $1 \alpha$ band from $n=5$ independent sets of animals. Data are expressed relative to wild-type (WT) hypoxic preconditioned (HPC) animals (=100\%). No significant difference between WT HPC and GPx1 transgenic HPC by one-way ANOVA.
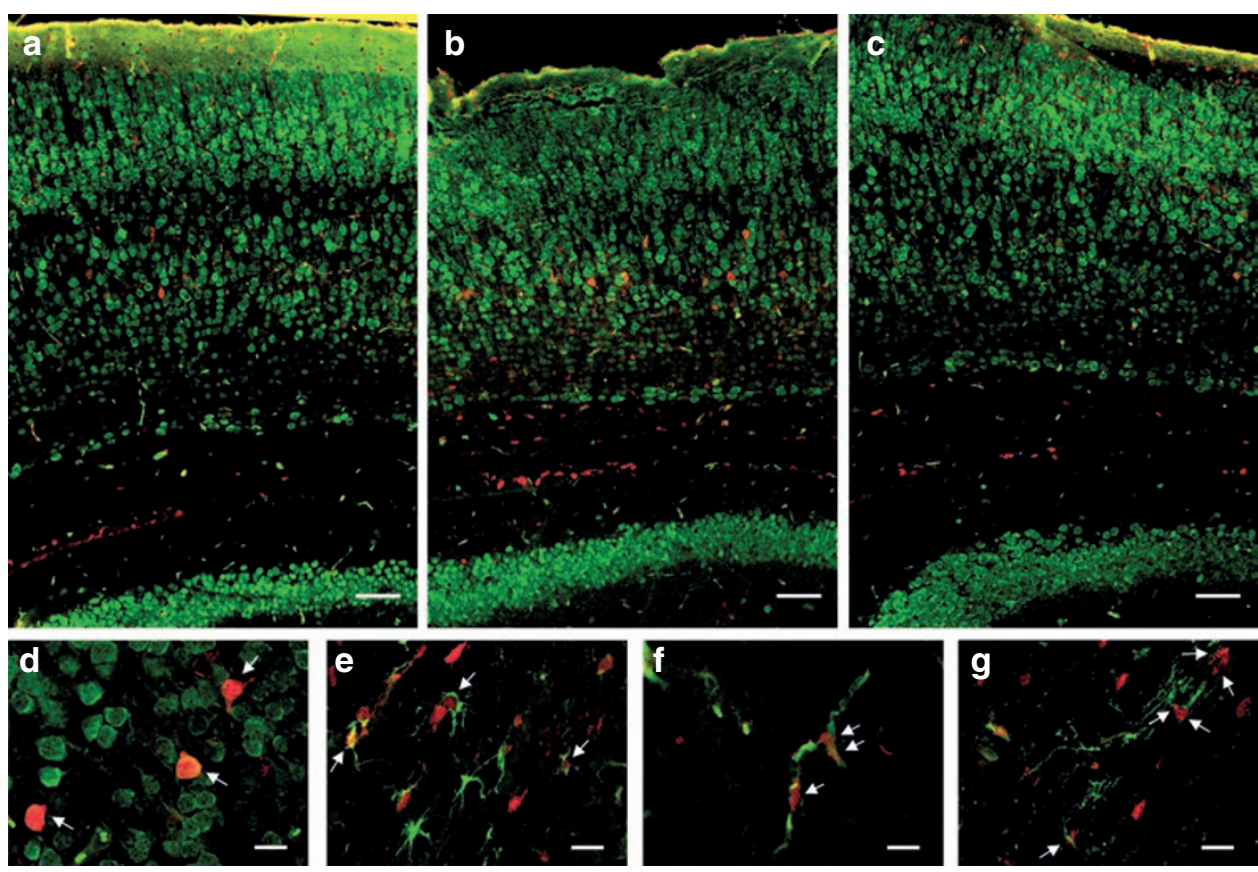

Figure 4. Cellular localization of activated extracellular-regulated kinase (ERK) in the cortex at $0.5 \mathrm{~h}$ posthypoxia. Coronal sections were stained for phospho-ERK (pERK) in red and neuronal nuclear antigen (NeuN), isolectin B4 (IB4), glial fibrillary acidic protein (GFAP), or 2', 3'-cyclic nucleotide $3^{\prime}$-phosphodiesterase (CNPase) in green. (a-c) Overviews of stitched images taken at 20x original magnification of (a) wild-type (WT) naive, (b) WT preconditioned, and (c) glutathione peroxidase 1 transgenic preconditioned stained for pERK and NeuN. Images are representative of at least $n=3$ independent animals. Scale bars represent $100 \mu \mathrm{m}$. (d-g) Colocalization of pERK with cellular markers (d) NeuN, (e) GFAP, (f) IB4, and (g) CNPase in sections from preconditioned WT animals $(0.5 \mathrm{~h}$ posthypoxia) at higher magnification (40x original magnification). Arrows point to (d) pERK-positive neurons, (e) astrocytes, (f) endothelial cells, and (g) oligodendrocytes. Scale bars represent $25 \mu \mathrm{m}$. 


\section{Articles $\mid$ Autheman et al.}

$\sim 50 \%$ below prehypoxic levels at $24 \mathrm{~h}$ after HPC, whereas those in WT animals returned to baseline at this time point. In the cortex of preconditioned WT animals, enhanced pERK staining was primarily observed in neurons and to a lower extent in astrocytes and endothelial cells, and was prominently nuclear in appearance. In contrast to the effect on ERK activation, GPx 1 overexpression had no effect on the induction of HIF- $1 \alpha$.

Similar to the findings in a neonatal rat model (11), HPC in WT mice is accompanied by transient activation of ERK but not by activation of Akt in the cortex. In the study by Jones and Bergeron, pERK $1 / 2$ levels were significantly increased $0.5 \mathrm{~h}$ after HPC with $8 \%$ oxygen for $3 \mathrm{~h}$, while phosphorylation of c-Jun $\mathrm{N}$-terminal kinase, p38, Akt, and the downstream target of Akt, glycogen synthase kinase $3 \beta$, remained unchanged, indicating that Akt was also not important in their model. In their study, inhibition of the ERK1/2 upstream kinase MEK1/2 with U0126 at the beginning of the preconditioning phase significantly attenuated protection afforded by HPC, indicating that ERK signaling plays an important role in HPC-mediated protection from $\mathrm{HI}$ injury in the neonatal brain. Partial ERK dependence of HPC protection was also observed in primary cortical neurons, where hypoxic tolerance is attenuated by U0126 (18). Other preconditioning stimuli such as oxygen-glucose deprivation (OGD) were also shown to require ERK signaling in the development of neuronal tolerance (10). Administration of the MEK1/2 inhibitor PD98059 blocked the activation of ERK and the protection induced by the nonlethal OGD preconditioning stimulus. Ischemic preconditioning in a global ischemia model was associated with an early activation of ERK in the hippocampus within the first few minutes after reoxygenation (19), followed by a second, more pronounced ERK activation 1-3 d later. Intracerebroventricular infusion of PD98059 shortly before the second ischemia abolished the activation of ERK $3 \mathrm{~d}$ after preconditioning ischemia and reversed its protective effect (20). Thus, transient activation of ERK is an important feature of different paradigms of preconditioning protection. Although our main focus was on the cortex, we also saw activation of ERK in the hippocampus (by immunofluorescence), but apparently to a lesser degree than in the cortex. Although this appears to be different from models in which global ischemia is used as either preconditioning stimulus or used to induce injury, where robust activation of ERK has been reported (21), we are not aware of a region-specific protective effect of HPC in the neonatal $\mathrm{HI}$ model.

The reversal of HPC protection by GPx1 overexpression (2) strongly implicates reactive oxygen species (ROS) such as hydrogen peroxide in HPC. Several in vitro studies have shown that exposure of cultured neurons to ROS-generating systems and defined ROS, such as hydrogen peroxide, induce protection from subsequent injury and have shown that antioxidants inhibit ischemic preconditioning. Exposure of cultured chick embryo neurons to xanthine/xanthine oxidase (which primarily generates superoxide anion radicals) or ferrous sulfate (which generates hydroxyl radicals) protected the cells from excitotoxic injury induced by excessive glutamate, staurosporine-induced apoptosis, or oxidative injury (22). In another study, cultured rat cortical neurons were preconditioned with xanthine oxidase, hydrogen peroxide, or a short OGD stimulus before being subject to prolonged OGD (23). All of these treatments substantially protected from OGD-induced injury. Ischemic and xanthine/xanthine oxidase-induced preconditioning were blocked by the addition of exogenous catalase but not a superoxide dismutase mimetic, indicating that hydrogen peroxide is responsible for inducing protection. The preconditioning effect of hydrogen peroxide is observed only at relatively low concentrations, whereas higher concentrations further increased OGDinduced injury. In a focal ischemia-reperfusion model in rats, ischemic preconditioning protection was abolished by treatment with the antioxidant and glutathione precursor $\mathrm{N}$-acetylcysteine and robust preconditioning could be achieved by direct infusion of hydrogen peroxide into the cortex (24). However, these studies did not look at the signaling mechanisms involved in preconditioning protection.

In this study, we show that GPx1 overexpression prevents the activation of ERK but not the induction of HIF-1 $\alpha$. Hydrogen peroxide at low (but not high) doses has been shown to induce HIF- $1 \alpha$ in primary cortical neurons, which was associated with protection against OGD (25). It is known from in vitro experiments that mitochondrial ROS play an important role in the induction of HIF- $1 \alpha$ by hypoxia (15). This contrasts with our observation of the GPx1 overexpressors, in which HIF-1 $\alpha$ induction was unaffected. Although we have no explanation for the discrepancy, it might be related to the fact that our experiments were conducted in vivo, where oxygen concentrations are much lower ( $5 \%$ in the brain) than in cultured cells kept at $21 \%$ oxygen. Cultivation of cells at ambient air is known to be associated with lowered expression of the different prolyl hydroxylases responsible for degradation/stabilization of HIF-1 $\alpha$ (26). The redox dependence of ERK activation has been reported in several in vitro models of oxidative stress. Indeed, hydrogen peroxide exposure stimulates rapid and transient phosphorylation of ERK in several cell types, including neurons, which protects them from subsequent oxidative injury (13). ERK is activated in cultured hippocampal neurons following oxidative injury as part of the cellular response to oxidative stress (27). Although the study showed that early ERK activation is involved in protection against glutamate-induced toxicity, late activation of ERK appears to be involved in toxicity. In a global ischemia preconditioning model, administration of the antioxidant $\alpha$-tocopherol attenuated ERK activation during postischemic reperfusion in the hippocampus, also linking ERK activation to ROS production (28). Early ERK activation postischemia has been shown to be related to the ROS- and Src-dependent activation of Raf-1 $(28,29)$. Our in vivo results with GPx1 tg mice clearly show that it is hydrogen peroxide that mediates the activation of neuroprotective ERK signaling by HPC and that aberrant ERK activation can explain the paradoxical reversal of HPC protection in GPx1 tg mice (2).

Induction of HIF- $1 \alpha$ has been suggested to play an important role in HPC-mediated protection of the neonatal rat brain from HI injury (30). Thus, protection by HPC was associated with marked induction of HIF-1 $\alpha$ and was mimicked 
by the application of chemical inducers of HIF-1 $1 \alpha$. However, neuron-specific knockout of HIF- $1 \alpha$ in mice does not inhibit the protective effects of HPC toward brain injury in a transient focal ischemia model, indicating that HIF- $1 \alpha$ is not absolutely required for preconditioning protection (31). The beneficial role of HIF-1 $\alpha$ in ischemic injury is also not quite clear. Although neuron-specific knockout of HIF- $1 \alpha$ causes less brain injury in a global ischemia model (32), neuronspecific knockout of HIF- $1 \alpha$ is associated with increased brain injury and decreased survival after transient focal ischemia (31) and with increased brain injury after neonatal HI (33). Although we have shown in this study that GPx1 overexpression does not affect HPC-mediated induction of HIF- $1 \alpha$, there exists a link between the transcriptional activity of HIF- $1 \alpha$ and activation of ERK. In human microvascular endothelial cells, it was shown that hypoxia-induced activation of ERK was required for transcriptional activity but not induction of HIF-1 $\alpha$ (34). ERK has been shown to phosphorylate HIF-1 $\alpha$ at Ser 641 and 643, thereby, increasing its nuclear retention and transcriptional activity (35). An involvement of ERK in the activation of HIF-1 $\alpha$ has recently also been demonstrated in a neonatal rat $\mathrm{HI}$ model in which induction of HIF- $1 \alpha$ and its target gene product vascular endothelial growth factor were partially blocked by inhibition of the ERK pathway (36).

We show here that HPC induces ERK activation primarily in pyramidal neurons in the cortex and to a lesser degree also in astrocytes and endothelial cells. The increased activation of ERK is localized to the nucleus. It is likely that activation of ERK induces a transcriptional response in these cells that makes them more resistant toward subsequent injury. Although activation of ERK seems to be involved in causing injury in the core of the ischemic infarct (37), activation in the penumbra may actually induce neuroprotective mechanisms similar to those induced by HPC or exogenous administration of neuroprotective growth factors such as brain-derived neurotrophic factor (38). As neuroprotective ERK signaling in our setting is clearly redox-dependent, and this may also be true for activation of neuroprotective ERK signaling after ischemic injury, treatment with antioxidants may not be the best treatment option for this type of injury.

\section{METHODS}

Animals

Heterozygous tg mice carrying the human GPxl gene were bred with CD1 WT mice to produce mixed litters of heterozygous GPx1overexpressing pups (GPx1 tg) and WT littermates as described previously $(4,39)$.

\section{HPC Stimulus}

Preconditioning hypoxia was carried out by placing pups in a chamber partially submerged in a $37^{\circ} \mathrm{C}$ water bath, through which $8 \%$ oxygen/ balance nitrogen flowed for $45 \mathrm{~min}$. One pup was monitored to ensure that rectal temperature did not exceed $37^{\circ} \mathrm{C}$. Control ("naive") pups were kept in a chamber exposed to room air. Mice were killed by rapid decapitation at $0,0.5,6,12$, and $24 \mathrm{~h}$ after reoxygenation and brains processed for the different analyses as described in the following. Animal experiments have been approved by the UCSF Institutional Animal Care and Use Committee and have been conducted according to National Institutes of Health guidelines.

\section{Whole-Cell Extracts}

The cortex was removed from the rest of the brain on a cold pack, and left and right cortex hemispheres flash frozen on dry ice and stored at $-80^{\circ} \mathrm{C}$ until analysis. Whole-cell extracts were prepared as described before (40). Briefly, one cortex hemisphere was homogenized in 1:10 modified radio-immunoprecipitation assay buffer containing $0.1 \%$ sodium dodecyl sulfate and the following protease and phosphatase inhibitors: Aprotinin $1 \mu \mathrm{g} / \mathrm{ml}$ (Sigma, St Louis, MO), Leupeptin $1 \mu \mathrm{g} / \mathrm{ml}$ (Sigma), Pepstatin $1 \mu \mathrm{g} / \mathrm{ml}$ (Sigma), dithiotreitol (Sigma), phenylmethylsulfonyl fluoride $1 \mathrm{mmol} / \mathrm{l}$ (Calbiochem, San Diego, CA), $1 \times$ Phosphatase Inhibitor Cocktail 1 (Sigma), sodium orthovanadate $1 \mathrm{mmol} / 1$ (Sigma), sodium fluoride $1 \mathrm{mmol} / 1$ (Sigma), $\beta$-glycerophosphate $2 \mathrm{mmol} / \mathrm{l}$ (Calbiochem), and sodium pyrophosphate $20 \mathrm{mmol} / \mathrm{l}$ (Sigma). The protein concentration of the whole cell extract was determined using a BCA assay kit (Sigma).

\section{Nuclear Extracts}

GPx1 tg and WT mice were killed by decapitation and then immediately perfused with phosphate buffered saline (PBS) containing $1 \%$ Phosphatase Inhibitor Cocktail 3 (Sigma). Cortices were dissected in cold PBS supplemented with 1\% Phosphatase Inhibitor Cocktail 3 (Sigma) and immediately frozen on dry ice. Brains from naive postnatal day 7 littermates were used as controls. Nuclear extracts were prepared as described $(41,42)$ using the NE-PER extraction kit from Pierce (Rockford, IL). Reagents were supplemented with protease and phosphatase inhibitors as mentioned for whole-cell extracts. Protein concentration was measured with Bradford assay (Sigma).

\section{Western Blotting}

Western blotting of phosphorylated proteins was performed as described previously (40). Equal amounts of brain tissue proteins $(80 \mu \mathrm{g}$ for whole-cell extracts and $10 \mu \mathrm{g}$ for nuclear extracts) were loaded on reducing $10 \%$ sodium dodecyl sulfate polyacrylamide gels and separated by electrophoresis. After blotting of the proteins on a nitrocellulose membrane (Whatman Optitran reinforced nitrocellulose

Table 1. Primary antibodies and lectins used in this study

\begin{tabular}{|c|c|c|c|}
\hline Antibody & Clone & $\begin{array}{l}\text { Catalog } \\
\text { number }\end{array}$ & Dilution \\
\hline $\begin{array}{l}\text { Rabbit monoclonal } \\
\text { antiphospho-ERK1/2 } \\
\text { (Thr202/Tyr204) }\end{array}$ & D13.14.4E & CST 4370 & $\begin{array}{l}1: 2,000 \text { (WB); } \\
\text { 1:200 (IF) }\end{array}$ \\
\hline $\begin{array}{l}\text { Rabbit monoclonal } \\
\text { antiphospho-Akt Ser473 }\end{array}$ & $193 \mathrm{H} 12$ & CST 4058 & $1: 1,000$ (WB) \\
\hline $\begin{array}{l}\text { Rabbit polyclonal } \\
\text { anti-ERK1/2 }\end{array}$ & & CST 9102 & $1: 1,000$ (WB) \\
\hline Rabbit polyclonal anti-Akt & & CST 9272 & $1: 1,000$ (WB) \\
\hline $\begin{array}{l}\text { Rabbit polyclonal } \\
\text { antimouse HIF-1 } \alpha\end{array}$ & & NB100-479 & $1: 1,000$ (WB) \\
\hline $\begin{array}{l}\text { Mouse monoclonal } \\
\text { anti-HIF-1 } \beta\end{array}$ & $\mathrm{H} 1 \beta 234$ & NB100-124 & $1: 500$ (WB) \\
\hline $\begin{array}{l}\text { Mouse monoclonal } \\
\text { anti- } \beta \text {-actin }\end{array}$ & AC-74 & A5316 & $1: 20,000(\mathrm{WB})$ \\
\hline $\begin{array}{l}\text { Mouse monoclonal } \\
\text { antineuronal nuclei }\end{array}$ & A60 & MAB377 & $1: 200$ (IF) \\
\hline $\begin{array}{l}\text { Mouse monoclonal antiglial } \\
\text { fibrillary acidic protein }\end{array}$ & GA5 & MAB360 & $1: 500$ (IF) \\
\hline $\begin{array}{l}\text { Mouse monoclonal } \\
\text { anti-2', } 3^{\prime} \text {-cyclic nucleotide } \\
3^{\prime} \text {-phosphodiesterase }\end{array}$ & $11-5 B$ & MAB326 & $1: 200$ (IF) \\
\hline $\begin{array}{l}\text { Isolectin IB4, Alexa Fluor } 488 \\
\text { conjugate }\end{array}$ & & 121411 & $1: 500$ (IF) \\
\hline
\end{tabular}

A, Sigma; CST, Cell Signaling Technology; ERK, extracellular-regulated kinase; HIF,

hypoxia-inducible factor; I, invitrogen; IB4, isolectin B4; IF, immunofluorescence; $M A B$,

Chemicon; NB, Novus Biological; WB, western blotting. 
membrane, pore size $0.45 \mu \mathrm{m}, \mathrm{GE}$ Healthcare, Waukesha, WI), nonspecific binding sites were blocked with $5 \%$ nonfat dry milk in Trisbuffered saline containing $0.1 \%$ tween. Primary antibodies listed in Table 1 were used at the dilution indicated and incubated overnight at $4{ }^{\circ} \mathrm{C}$. Horseradish peroxidase-conjugated mouse antirabbit or rabbit antimouse (both from Sigma, 1:20,000) were used as secondary antibodies. Membranes were stripped with Restore Western blot stripping buffer (Pierce) and reprobed with a different primary antibody several times. To verify equal protein loading, blots were probed with a mouse monoclonal anti- $\beta$-actin antibody (Sigma, 1:10,000). Immunoreactive bands were detected using Immobilon Western Chemiluminescent HRP Substrate from Millipore (Billerica, MA) and acquired using FluorChem SP with AlphaEaseFC software (Alpha Innotech, San Leandro, CA).

\section{Colocalization of pERK With Cell Type-Specific Markers}

Double-immunofluorescence staining of $6 \mu \mathrm{m}$ paraffin sections was performed as described previously (40). Briefly, mice were killed by cervical dislocation $30 \mathrm{~min}$ after the end of hypoxia and immediately perfused with cold PBS containing 1\% Phosphatase Inhibitor Cocktail 3 and sodium orthovadanate $1 \mathrm{mmol} / \mathrm{l}$ (Sigma). Brains were removed from the skull and fixed for $24 \mathrm{~h}$ in $4 \%$ phosphate-buffered paraformaldehyde containing 1\% PhosStop (Roche, Mannheim, Germany). Fixed brains were then transferred to $50 \%$ ethanol before paraffin embedding and cut into $6 \mu \mathrm{m}$ coronal sections using a microtome. Three regions of the cortex (anterior, middle, and posterior) and the hippocampus were analyzed. Sections were deparaffinized in xylol, rehydrated in increasing concentration of ethanol, and boiled for $10 \mathrm{~min}$ in $10 \mathrm{mmol} / \mathrm{l}$ sodium citrate $\mathrm{pH} 6$ for antigen retrieval (43). Sections were blocked for $2 \mathrm{~h}$ in PBS/Triton X-100 0.3\% containing $10 \%$ normal goat serum. Primary antibodies (Table 1) were diluted in PBS/Triton X-100 0.3\% containing $1 \%$ bovine serum albumin and $2 \%$ normal goat serum and incubated overnight at $4{ }^{\circ} \mathrm{C}$. Sections were then incubated for $2 \mathrm{~h}$ with secondary antibodies Alexa 555 goat antirabbit IgG and Alexa 488 goat antimouse IgG (Invitrogen, Carlsbad, CA) diluted in PBS/Triton X-100 0.3\% at 1:1,000. Sections were counterstained with 4', 6'-diamidino-2-phenylindol and mounted with Prolong Gold Antifade Reagent (Invitrogen). Images were acquired on a wide-field epifluorescence microscope (Axio Imager M1, Carl Zeiss Micro Imaging, Jena, Germany) using AxioVision software (Carl Zeiss Micro Imaging). The specificity of the antibody directed against pERK was assessed by incubating sections without primary antibody or with normal rabbit serum.

\section{Data Analysis}

Digitized western blots were analyzed densitometrically using AlphaView Software (Alpha Innotech) and are expressed as percentage relative to respective naive mice or to hypoxia-treated WT mice for HIF- $1 \alpha$ stabilization measurement. Results are expressed as mean \pm SEM and statistical analysis was performed using Prism 5 (GraphPad, San Diego, CA). Student's $t$-test was used for comparison between two groups and one-way ANOVA with Dunnett's post hoc test for comparison between multiple groups. Two-way ANOVA was used to compare the kinetics of ERK activation in WT vs. GPx1 tg mice. Differences were considered to be statistically significant at $P<0.05$.

\section{STATEMENT OF FINANCIAL SUPPORT}

This study was supported by National Institute of Neurological Disorders and Stroke R01 grant NS33997 (to D.M.F. and S.C.) and Swiss National Science Foundation grant 3100-120725 (to S.C.).

\section{REFERENCES}

1. Dirnagl U, Becker K, Meisel A. Preconditioning and tolerance against cerebral ischaemia: from experimental strategies to clinical use. Lancet Neurol 2009;8:398-412.

2. Sheldon RA, Aminoff A, Lee CL, Christen S, Ferriero DM. Hypoxic preconditioning reverses protection after neonatal hypoxia-ischemia in glutathione peroxidase transgenic murine brain. Pediatr Res 2007;61:666-70.

3. Fullerton HJ, Ditelberg JS, Chen SF, et al. Copper/zinc superoxide dismutase transgenic brain accumulates hydrogen peroxide after perinatal hypoxia ischemia. Ann Neurol 1998;44:357-64.
4. Sheldon RA, Jiang X, Francisco C, et al. Manipulation of antioxidant pathways in neonatal murine brain. Pediatr Res 2004;56:656-62.

5. Weisbrot-Lefkowitz $\mathrm{M}$, Reuhl $\mathrm{K}$, Perry $\mathrm{B}$, Chan $\mathrm{PH}$, Inouye $\mathrm{M}$, Mirochnitchenko O. Overexpression of human glutathione peroxidase protects transgenic mice against focal cerebral ischemia/reperfusion damage. Brain Res Mol Brain Res 1998;53:333-8.

6. Sheldon RA, Christen S, Ferriero DM. Genetic and pharmacologic manipulation of oxidative stress after neonatal hypoxia-ischemia. Int J Dev Neurosci 2008;26:87-92.

7. Ditelberg JS, Sheldon RA, Epstein CJ, Ferriero DM. Brain injury after perinatal hypoxia-ischemia is exacerbated in copper/zinc superoxide dismutase transgenic mice. Pediatr Res 1996;39:204-8.

8. Sawe N, Steinberg G, Zhao H. Dual roles of the MAPK/ERK1/2 cell signaling pathway after stroke. J Neurosci Res 2008;86:1659-69.

9. Wick A, Wick W, Waltenberger J, Weller M, Dichgans J, Schulz JB. Neuroprotection by hypoxic preconditioning requires sequential activation of vascular endothelial growth factor receptor and Akt. J Neurosci 2002;22:6401-7.

10. Gonzalez-Zulueta M, Feldman AB, Klesse LJ, et al. Requirement for nitric oxide activation of p21(ras)/extracellular regulated kinase in neuronal ischemic preconditioning. Proc Natl Acad Sci USA 2000;97:436-41.

11. Jones NM, Bergeron M. Hypoxia-induced ischemic tolerance in neonatal rat brain involves enhanced ERK1/2 signaling. J Neurochem 2004;89:157-67.

12. Zhao H, Sapolsky RM, Steinberg GK. Phosphoinositide-3-kinase/akt survival signal pathways are implicated in neuronal survival after stroke. Mol Neurobiol 2006;34:249-70.

13. Guyton KZ, Liu Y, Gorospe M, Xu Q, Holbrook NJ. Activation of mitogenactivated protein kinase by $\mathrm{H} 2 \mathrm{O} 2$. Role in cell survival following oxidant injury. J Biol Chem 1996;271:4138-42.

14. Leslie NR. The redox regulation of PI 3-kinase-dependent signaling. Antioxid Redox Signal 2006;8:1765-74.

15. Chandel NS, McClintock DS, Feliciano CE, et al. Reactive oxygen species generated at mitochondrial complex III stabilize hypoxia-inducible factor- $1 \alpha$ during hypoxia: a mechanism of $\mathrm{O}_{2}$ sensing. $\mathrm{J}$ Biol Chem 2000;275:25130-8.

16. Sheldon RA, Sedik C, Ferriero DM. Strain-related brain injury in neonatal mice subjected to hypoxia-ischemia. Brain Res 1998;810:114-22.

17. Mebratu Y, Tesfaigzi Y. How ERK1/2 activation controls cell proliferation and cell death: is subcellular localization the answer? Cell Cycle 2009;8:1168-75

18. Ma MC, Qian H, Ghassemi F, Zhao P, Xia Y. Oxygen-sensitive $\{\Delta\}$-opioid receptor-regulated survival and death signals: novel insights into neuronal preconditioning and protection. J Biol Chem 2005;280:16208-18.

19. Gu Z, Jiang Q, Zhang G, Cui Z, Zhu Z. Diphosphorylation of extracellular signal-regulated kinases and c-Jun $\mathrm{N}$-terminal protein kinases in brain ischemic tolerance in rat. Brain Res 2000;860:157-60.

20. Gu Z, Jiang Q, Zhang G. Extracellular signal-regulated kinase and c-Jun N-terminal protein kinase in ischemic tolerance. Neuroreport 2001;12:3487-91.

21. Choi JS, Kim HY, Cha JH, Lee MY. Ischemic preconditioning-induced activation of ERK1/2 in the rat hippocampus. Neurosci Lett 2006;409:187-91.

22. Ravati A, Ahlemeyer B, Becker A, Krieglstein J. Preconditioning-induced neuroprotection is mediated by reactive oxygen species. Brain Res 2000;866:23-32.

23. Furuichi T, Liu W, Shi H, Miyake M, Liu KJ. Generation of hydrogen peroxide during brief oxygen-glucose deprivation induces preconditioning neuronal protection in primary cultured neurons. J Neurosci Res 2005;79:816-24.

24. Simerabet M, Robin E, Aristi I, et al. Preconditioning by an in situ administration of hydrogen peroxide: involvement of reactive oxygen species and mitochondrial ATP-dependent potassium channel in a cerebral ischemiareperfusion model. Brain Res 2008;1240:177-84.

25. Chang S, Jiang X, Zhao C, Lee C, Ferriero DM. Exogenous low dose hydrogen peroxide increases hypoxia-inducible factor-1alpha protein expression and induces preconditioning protection against ischemia in primary cortical neurons. Neurosci Lett 2008;441:134-8. 
26. Khanna S, Roy S, Maurer M, Ratan RR, Sen CK. Oxygen-sensitive reset of hypoxia-inducible factor transactivation response: prolyl hydroxylases tune the biological normoxic set point. Free Radic Biol Med 2006;40: 2147-54.

27. Luo Y, DeFranco DB. Opposing roles for ERK1/2 in neuronal oxidative toxicity: distinct mechanisms of ERK1/2 action at early versus late phases of oxidative stress. J Biol Chem 2006;281:16436-42.

28. Wu HW, Li HF, Wu XY, Zhao J, Guo J. Reactive oxygen species mediate ERK activation through different Raf-1-dependent signaling pathways following cerebral ischemia. Neurosci Lett 2008;432:83-7.

29. Guo J, Wu HW, Hu G, Han X, De W, Sun YJ. Sustained activation of Srcfamily tyrosine kinases by ischemia: a potential mechanism mediating extracellular signal-regulated kinase cascades in hippocampal dentate gyrus. Neuroscience 2006;143:827-36.

30. Bergeron M, Gidday JM, Yu AY, Semenza GL, Ferriero DM, Sharp FR. Role of hypoxia-inducible factor-1 in hypoxia-induced ischemic tolerance in neonatal rat brain. Ann Neurol 2000;48:285-96.

31. Baranova O, Miranda LF, Pichiule P, Dragatsis I, Johnson RS, Chavez JC. Neuron-specific inactivation of the hypoxia inducible factor 1 alpha increases brain injury in a mouse model of transient focal cerebral ischemia. J Neurosci 2007;27:6320-32.

32. Helton R, Cui J, Scheel JR, et al. Brain-specific knock-out of hypoxiainducible factor-1alpha reduces rather than increases hypoxic-ischemic damage. J Neurosci 2005;25:4099-107.

33. Sheldon RA, Osredkar D, Lee CL, Jiang X, Mu D, Ferriero DM. HIF-1 alpha-deficient mice have increased brain injury after neonatal hypoxiaischemia. Dev Neurosci 2009;31:452-8.

34. Minet E, Arnould T, Michel G, et al. ERK activation upon hypoxia: involvement in HIF-1 activation. FEBS Lett 2000;468:53-8.
35. Mylonis I, Chachami G, Samiotaki M, et al. Identification of MAPK phosphorylation sites and their role in the localization and activity of hypoxiainducible factor-1alpha. J Biol Chem 2006;281:33095-106.

36. Li L, Xiong Y, Qu Y, et al. The requirement of extracellular signal-related protein kinase pathway in the activation of hypoxia inducible factor 1 alpha in the developing rat brain after hypoxia-ischemia. Acta Neuropathol 2008;115:297-303.

37. Wang X, Zhu C, Qiu L, Hagberg H, Sandberg M, Blomgren K. Activation of ERK1/2 after neonatal rat cerebral hypoxia-ischaemia. J Neurochem 2003;86:351-62.

38. Han BH, Holtzman DM. BDNF protects the neonatal brain from hypoxicischemic injury in vivo via the ERK pathway. J Neurosci 2000;20:5775-81.

39. Mirault ME, Tremblay A, Furling D, et al. Transgenic glutathione peroxidase mouse models for neuroprotection studies. Ann N Y Acad Sci 1994;738:104-15.

40. Sury MD, Agarinis C, Widmer HR, Leib SL, Christen S. JNK is activated but does not mediate hippocampal neuronal apoptosis in experimental neonatal pneumococcal meningitis. Neurobiol Dis 2008;32:142-50.

41. Sury MD, Frese-Schaper M, Mühlemann MK, et al. Evidence that $\mathrm{N}$-acetylcysteine inhibits TNF-alpha-induced cerebrovascular endothelin-1 upregulation via inhibition of mitogen- and stress-activated protein kinase. Free Radic Biol Med 2006;41:1372-83.

42. Lahiri DK, Ge Y. Electrophoretic mobility shift assay for the detection of specific DNA-protein complex in nuclear extracts from the cultured cells and frozen autopsy human brain tissue. Brain Res Brain Res Protoc 2000;5:257-65.

43. Tang X, Falls DL, LiX, Lane T, Luskin MB. Antigen-retrieval procedure for bromodeoxyuridine immunolabeling with concurrent labeling of nuclear DNA and antigens damaged by $\mathrm{HCl}$ pretreatment. J Neurosci 2007;27:5837-44. 